\title{
Effects of the mycotoxins Iolitrem $B$ and paxilline on gastrointestinal smooth muscle, the cardiovascular and respiratory systems, and temperature in sheep
}

\author{
L.M. McLEAY ${ }^{1}$ and B.L. SMITH ${ }^{2}$ \\ ${ }^{1}$ Department of Biological Sciences, University of Waikato, Private Bag 3105, Hamilton \\ ${ }^{2}$ AgResearch, Ruakura Research Centre, Private Bag 3123, Hamilton \\ ${ }^{1}$ L.McLeay@waikato.ac.nz
}

\begin{abstract}
The mycotoxins lolitrem B (responsible for ryegrass-staggers), and paxilline have a common moiety and are well known for their ability to cause tremoring in animals. Little is known of their other effects in ruminants and we investigated these in sheep, on electromyographic (EMG) activity of gastrointestinal smooth muscle of the reticulum, rumen, abomasal antrum and duodenum and on blood pressure, heart rate, respiration and body temperature. Both mycotoxins induced tremor and caused marked increases in respiratory rate, heart rate and blood pressure, but had relatively little effect on body temperature. Increases in blood pressure preceded substantial rises in heart rate, indicating a peripheral vasoconstrictive effect. Both mycotoxins had variable excitatory and inhibitory effects on the antrum and duodenum, but had consistent and profound effects on activity of the reticulorumen with a similar time course to their characteristic effects for paxilline and lolitrem B respectively, on the induction ( 2 and 30 minutes) and the duration (2-3 h and 8-12 h) of tremoring. The effects included an inhibition of the vagallydependent cyclical A and B sequences of contraction of the reticulorumen and an excitation of intrinsic activity including "chaotic" activity of the reticulum. Excitatory effects of the tremorgens were partially blocked by atropine, indicating some but not all, excitatory effects on the reticulorumen arise from stimulation of muscarinic cholinoceptors and it is possible this and other excitatory effects may be mediated through calcium-activated high conductance potassium channels. It is concluded tremorgens have widespread effects on the respiratory, cardiovascular and digestive systems. The intensity and duration of the effects with lolitrem B, indicate substantial disruption of digestion may occur with its ingestion on grazing endophyte-infected pasture.
\end{abstract}

Keywords: cardiovascular, electromyograph activity, endophyte, gastrointestinal, lolitrem B, mycotoxins, paxilline, respiration, reticulorumen, temperature

\section{Introduction}

Certain fungi are capable of producing toxins which elicit a sustained or intermittent tremoring in animals and among these are the lolitrems and paxilline which have been formally classified in a group based on their structural characteristics of a common indole nucleus linked to a diterpenoid unit (Steyn \& Vleggaar 1985). Lolitrem B is responsible for ryegrass staggers in livestock (Gallagher et al. 1981, 1984, Hawkes et al. 1993), but its activities have not been studied extensively, because of its short supply due to difficulties in its extraction and purification. Paxilline produced both by Penicillium species of fungi (Steyn \& Vleggaar 1985) and Neotyphodium lolii (Weedon \& Mantle 1987) is thought to be a precusor in the biosynthesis of lolitrem B by Neotyphodium lolii (Penn \& Mantle 1994; MundayFinch et al. 1995). Paxilline is more readily available, causes similar but shorter-lasting clinical signs in animals, and provides a valuable comparison in studies on the lesser known activities of lolitrem B. While most studies in ruminants have been related to tremoring, we have investigated the effects of paxilline and lolitrem $B$ on smooth muscle of the stomach and intestine (Smith et al. 1997; McLeay et al. 1999) and on other physiological activities including blood pressure, heart rate, respiration and body temperature.

\section{Materials and methods}

\section{Tremorgens}

Lolitrem B and paxilline were prepared by the methods described in McLeay et al. (1999). Both tremorgens were dissolved in acetone $(2 \mathrm{ml})$ and dose rates chosen $(0.66-1.5 \mathrm{mg} / \mathrm{kg}$ for paxilline and $25-110 \mu \mathrm{g} / \mathrm{kg}$ for lolitrem B) that produced moderate tremors in sheep (Smith et al. 1997).

\section{Animals}

Eight animals were used in the experiments on gastrointestinal smooth muscle and full details of the 
methods have been previously described (Smith et al. 1997; McLeay et al. 1999). Briefly, electromyograph (EMG) activity was recorded from electrodes surgically implanted in the reticulum, cranial dorsal rumen, abomasal antrum and duodenum. The EMG activity was integrated and used as an index of motility. For the experiments on other physiological functions, three castrated male Romney x Dorset sheep 9 months old (30-35 kg) were maintained indoors in pens on a daily diet of $1200 \mathrm{~g}$ dried chaffed meadow hay and $100 \mathrm{~g}$ concentrate pellets. Each sheep was prepared under general anaesthesia with a catheter inserted via a branch of the femoral artery, so that the tip lay in the abdominal aorta. The catheter was tunnelled subcutaneously and exteriorised in the mid-lumbar region. It was filled with heparinized saline $(5 \mathrm{IU} / \mathrm{ml})$ and usually flushed at twoday intervals. Such preparations were maintained for at least six months without blockage. At the start of each experiment the catheter was flushed with sterile saline and connected to a physiological pressure transducer (Bell and Howell Ltd, Basingstoke, England) and preamplifier (3552, Devices UK), for the recording of blood pressure. The transducer was mounted at the estimated level of the abdominal aorta in the sheep's standing position.

Before each tremorgen challenge, each sheep had three platinum cap electrodes (Devices UK) sutured to the skin over the left shoulder muscles which had previously been shown to produce good electromyographic recordings (Smith et al. 1997). Contact of these electrodes with the skin was aided by electrode jelly. The electrodes from both skeletal muscle and gut smooth muscle were connected to AC preamplifiers (Grass P15) and the EMGs integrated using a Devices 3520 integrator as previously described (McLeay et al. 1990).

In addition to EMG activity, movement of each animal's head was monitored over the experimental period with an Actiwatch Activity Monitoring System (Mini-Mitter Co., Inc. Sunriver OR, USA). The actiwatch was secured to the top of the head with velcro and on completion of the experiment the recording was downloaded to a PC computer for analysis.

The temperature of the animals was continuously recorded with a 20-gauge needle thermistor (Devices UK) inserted under local anaesthesia (2\% Xylocaine, Astra Pharmaceuticals Ltd, NSW, Australia), into a Longissimus dorsi muscle and connected to a preamplifier (3553, Devices UK), set at a range of 35$45^{\circ} \mathrm{C}$.

Respiration was recorded by strapping corrugated bellows (Stethograph/transducer, Harvard Apparatus Ltd, Kent, England), around the abdomen and connecting it to a physiological pressure transducer (Bell and Howell
Ltd, Basingstoke, England) and preamplifier (3552, Devices UK). The outputs from the preamplifiers for blood pressure, respiration and temperature and from the integrator for EMG activity were recorded on chart using an M19 chart recorder (Devices UK). Respiration rate and heart rate were measured from the continuous recording by running the chart at a faster speed for a 10second interval. In addition all outputs were captured on disc and analysed using a computer program (John Curtis, University of Waikato 1989) which provided total integrated volts under the trace over any time period.

At least 24 hours before each experiment, each sheep had an in-dwelling intravenous catheter inserted into its jugular vein for the administration of acetone and tremorgens. Animals were made to remain standing during experiments by suspending a sling beneath their brisket. Where tremors were prolonged, as in the case of those induced by lolitrem B, animals were permitted to lie 3.5 hours after giving lolitrem B and were stood up periodically for short intervals to record blood pressure and the presence or absence, of tremors.

\section{Experimental procedure}

Before each administration of tremorgen a normal recording was collected for at least 30 minutes followed by a further 30-minutes recording immediately after the administration of acetone and immediately before the administration of the tremorgen in the same volume of acetone. In addition, as separate controlled experiments, two consecutive administrations of $2 \mathrm{ml}$ of acetone, 30 minutes apart were given. The $2 \mathrm{ml}$ of infusate was given slowly over 2 minutes into the jugular catheter and washed in with $2 \mathrm{ml}$ heparinised saline. Recordings were continued for 3.5 hours after administration of the shorter acting paxilline and for 12 hours after administration of the longer acting lolitrem $B$ and for its controls. Each sheep received on separate days, control acetone, paxilline and lolitrem $B$ in a randomised block design.

These experiments were approved by The University of Waikato Animal Ethics Committee and the Ruakura Research Centre Animal Ethics Committee.

\section{Data analysis}

The effects of the substances on blood pressure, heart rate, respiration rate, temperature and EMG activity of skeletal and gut smooth muscles were observed for each sheep from the chart recordings and on head movement from the actiwatch records. Quantitative data on EMG activity provided using the computer program was normalised for each animal, by expressing EMG activity in volts over 15-minute periods, as a percentage of the average of the first two 15-minute periods. The maximal 
increases in heart rate, blood pressure, respiration rate and temperature above the control values before the first administration of acetone were averaged for the three sheep. The statistical significance of the mean maximum changes for each tremorgen, compared with the corresponding period of the acetone control was tested using Student's t-test. Clinical observations of all animals were recorded.

\section{Results}

Both tremorgens produced tremors and increased movement of the head as measured by the actiwatch and increased EMG activity of skeletal muscle. The effects of paxilline and lolitrem B on activity of skeletal muscle differ in that there is a very short latency ( 2 minutes) and relatively short longevity ( 2 hours) with paxilline, whereas with lolitrem $B$ the onset is delayed for about 30 minutes, but effects persisted for at least 12 hours, with the respective doses used here.

\section{Effects of paxilline and lolitrem B on EMG activity of gastrointestinal smooth muscle}

Paxilline: Paxilline was inhibitory on the antrum in two of the five animals, an effect which was immediate and lasted for up to one hour. A reduction in duodenal activity was obtained in four animals and in the other marked stimulation of phase III MMClike activity occurred.

Paxilline was both excitatory and inhibitory on the reticulum and rumen. The excitatory effects occurred within the 2minute infusion time and took the form of elevations in baseline of both the reticulum and rumen EMG and increases in the amplitude of the reticulum EMG associated with A sequences, both before and after, inhibitory responses occurred (Figure 1).

An inhibitory effect on A and B sequences of contraction became apparent soon after the elevation in baseline activity and these were completely abolished for up to about 1 hour (Figure 1). A and B sequences of contraction were maximally reduced in frequency (A sequences $\mathrm{P}<0.01$, $B$ sequences $\mathrm{P}<0.001$ ) from $30-60$ minutes and were reduced in frequency for a total period of about 2.5 hours (Figure 1).
Figure 1 Integrated electromyograms of the rumen (Ru) and reticulum (Ret) recorded over 4 hours in the same sheep during administration at 1 hour of (A) acetone control (Ace), (B) $1 \mathrm{mg} / \mathrm{kg}$ paxilline (Pax) and (C) $75 \mu \mathrm{g} / \mathrm{kg}$ lolitrem B (Lol B). The recordings are computer printouts of the stored data.

$\mathbf{R u}$

A

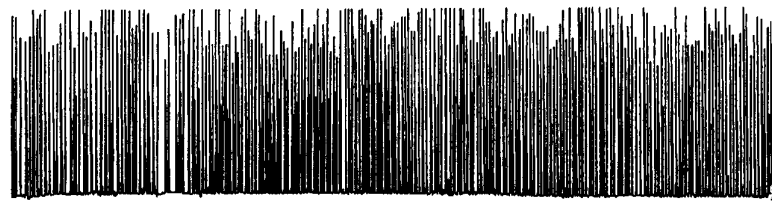

Ret

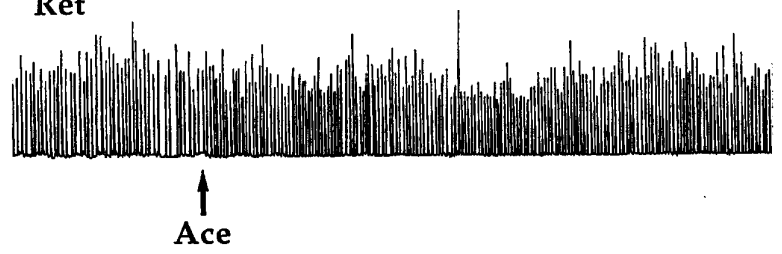

B
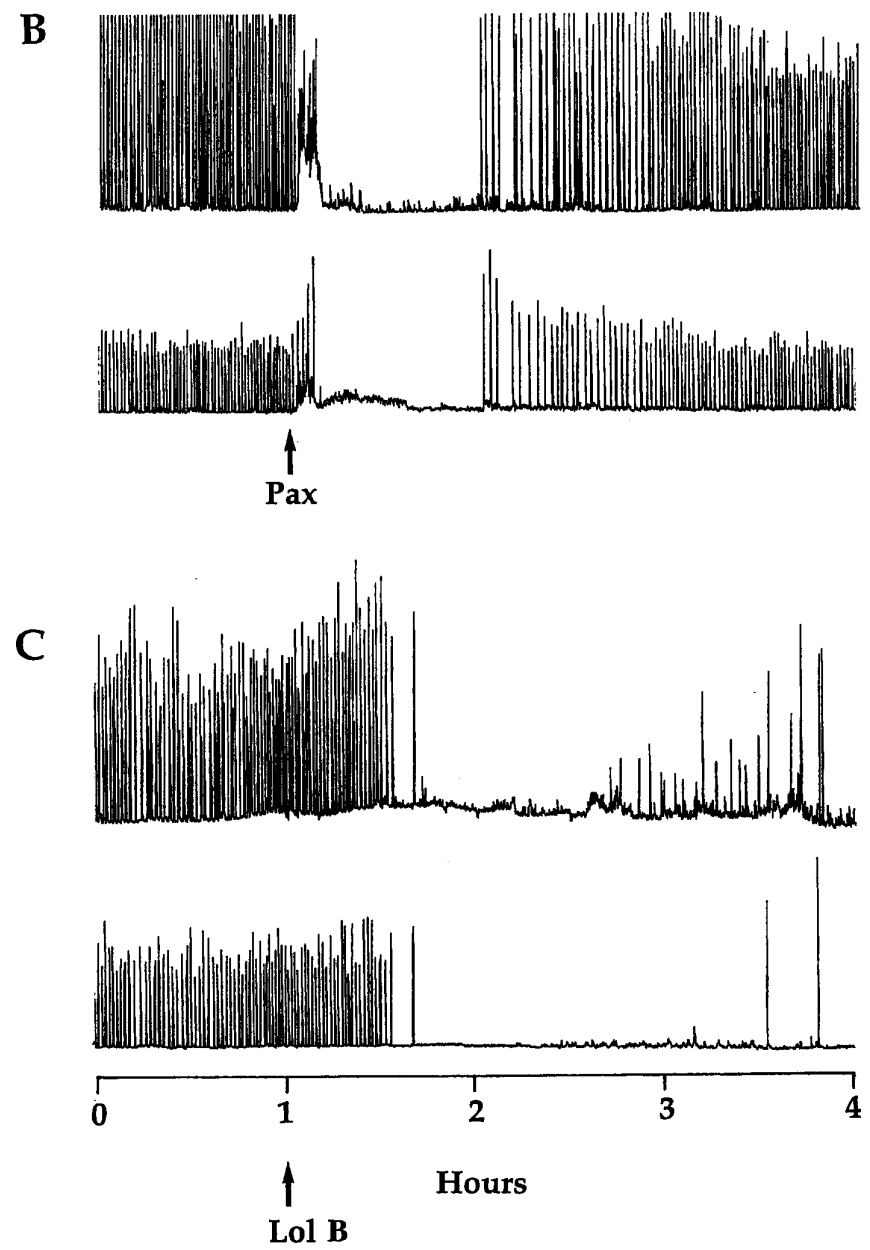
Lolitrem B.: Lolitrem B inhibited activity of the antrum in three sheep, the effect commenced within 30 minutes and continued for up to 2 hours. Duodenal activity was reduced in two animals and stimulated in a third animal. Lolitrem B inhibited the frequency of A and B sequences of contraction of the reticulum and rumen $(\mathrm{P}<0.001)$. Compared with paxilline the inhibition was delayed, becoming apparent after about 20-30 minutes (Figure 1) and was associated with the appearance of tremors . A and B sequences of contraction were virtually completely inhibited or markedly reduced in frequency (Figure 1) for up to 11-15 hours following administration of loltitrem B.

In two sheep an elevation in baseline EMG of the reticulum changed to activity which took the form of lower amplitude, faster frequency activity than that associated with A sequences (we have defined this as "chaotic" activity), and this marked excitatory activity was sustained for many hours during which time A and $\mathrm{B}$ sequences were abolished. The reappearance of $\mathrm{A}$ and B sequences after giving lolitrem B coincided with decreased "chaotic" activity and gradually normal motility and rumination returned after about 12 hours. In contrast to the reticulum, the rumen did not exhibit "chaotic" activity.

The muscarinic cholinoceptor blocker atropine (100 and $200 \mu \mathrm{g} / \mathrm{kg}$ i.v.) reduced dose-dependently, but did not abolish "chaotic" activity produced by lolitrem B and the intrinsic activity produced by paxilline.

\section{Effects of paxilline and lolitrem $B$ on blood pressure, heart rate, respiration and body temperature}

Both tremorgens induced tremor and caused marked increases in respiration rate, heart rate and blood pressure, but had relatively little effect on body temperature (Table 1).

Table 1 Maximal increases (means and standard deviations for 3 animals) in systolic and diastolic blood pressure, heart rate, respiration rate and body temperature after administration of acetone or tremorgens.

\begin{tabular}{lccccc}
\hline & Heart & Respiration & \multicolumn{2}{c}{- Blood Pressure --- } & Body \\
& Rate & Rate & Systolic & Diastolic & Temperature \\
\hline & No./min & No./min & $\mathrm{mm} \mathrm{Hg}$ & $\mathrm{mm} \mathrm{Hg}$ & ${ }^{\circ} \mathrm{C}$ \\
Acetone & 0 & $34 \pm 19$ & $4 \pm 17$ & $4 \pm 7$ & $0.17 \pm 0.31$ \\
Paxilline & $88 \pm 27^{* *}$ & $122 \pm 19^{* *}$ & $83 \pm 7^{* *}$ & $65 \pm 25^{*}$ & $0.73 \pm 1.10$ \\
Lolitrem B & $92 \pm 28^{* *}$ & $102 \pm 24^{*}$ & $52 \pm 26^{*}$ & $38 \pm 18^{*}$ & $1.13 \pm 0.85$ \\
\hline
\end{tabular}

* indicates $\mathrm{P}<0.05$, ** indicates $\mathrm{P}<0.01$, between acetone and tremorgens

Increases in blood pressure in animals given paxilline doubled to reach a maximum within 5 minutes in all three animals and gradually declined over the next two hours. In one animal heart rate had doubled by 10 minutes and gradually declined over the next two hours, showing a similar trend to blood pressure. In a second animal, heart rate remained stable and in the third animal, heart rate increased 1 hour after paxilline administration, at a time when blood pressure was dropping.

Blood pressure began to increase between 15 and 25 minutes after, and reached a maximum 1-1.5 hours after lolitrem B (Figure 2B). Thereafter blood pressure gradually declined, but remained above control levels for at least 7 hours (Figure 2B). Heart rate showed similar responses, but the rises and maxima reached were delayed by $30-60$ minutes, so that responses in blood pressure preceded those in heart rate (Figure 2B).

Respiration rate and body temperature increased in association with tremoring, which was short term (1-2 hours) with paxilline and long term (up to 7-8 hours) with lolitrem B (Figure 2A). Respiration rates in these animals were relatively high under control conditions, but increased further with administration of the tremorgens and took the form of high frequency, shallow depth panting.

\section{Discussion}

Paxilline and lolitrem B had consistent and profound excitatory and inhibitory effects on smooth muscle of the reticulum and rumen, whereas their effects on the abomasal antrum and duodenum were less marked and variable. In contrast to motility of the abomasum and duodenum, the cyclical A and B sequences of contractions of the reticulorumen are dependent upon the release of acetylcholine from the terminals of the vagus nerve (Titchen 1968). Previous work has indicated that excitatory effects of paxilline on smooth muscle of guinea pig intestine involved acetylcholine release (Selala et al. 1991). If the tremorgens influence neurotransmitter release or action, effects on motility of the reticulorumen should have been clearly evident and this was the case. The reduction in excitatory effects by the muscarinic cholinoceptor blocker atropine, is corroborative evidence for the involvement of acetylcholine in the responses. In terms of mechanisms for this action, paxilline has been shown to inhibit high-conductance calcium-activated $\mathrm{K}^{+}$channels (Knaus et al. 1994) and these have been shown to regulate neurotransmitter release (Robitallie \& Charlton 1992; Stretton et al. 1992). Consequently it has been argued that this is one possible mechanism for the excitatory effects of paxilline and lolitrem $B$ on the reticulorumen via an atropine-sensitive pathway (McLeay et al. 1999). 
Figure 2 Changes in physiological activities over 10 hours following the administration of control acetone (Ace.) at 30 minutes and $75 \mu \mathrm{g} /$ kg lolitrem B (Lol.) at 1.0 hours in Sheep 1. In A, tremor and movement ( $T$ \& M activity), heart rate, respiration rate and body temperature. In B, systolic and diastolic blood pressures and heart rate.

A

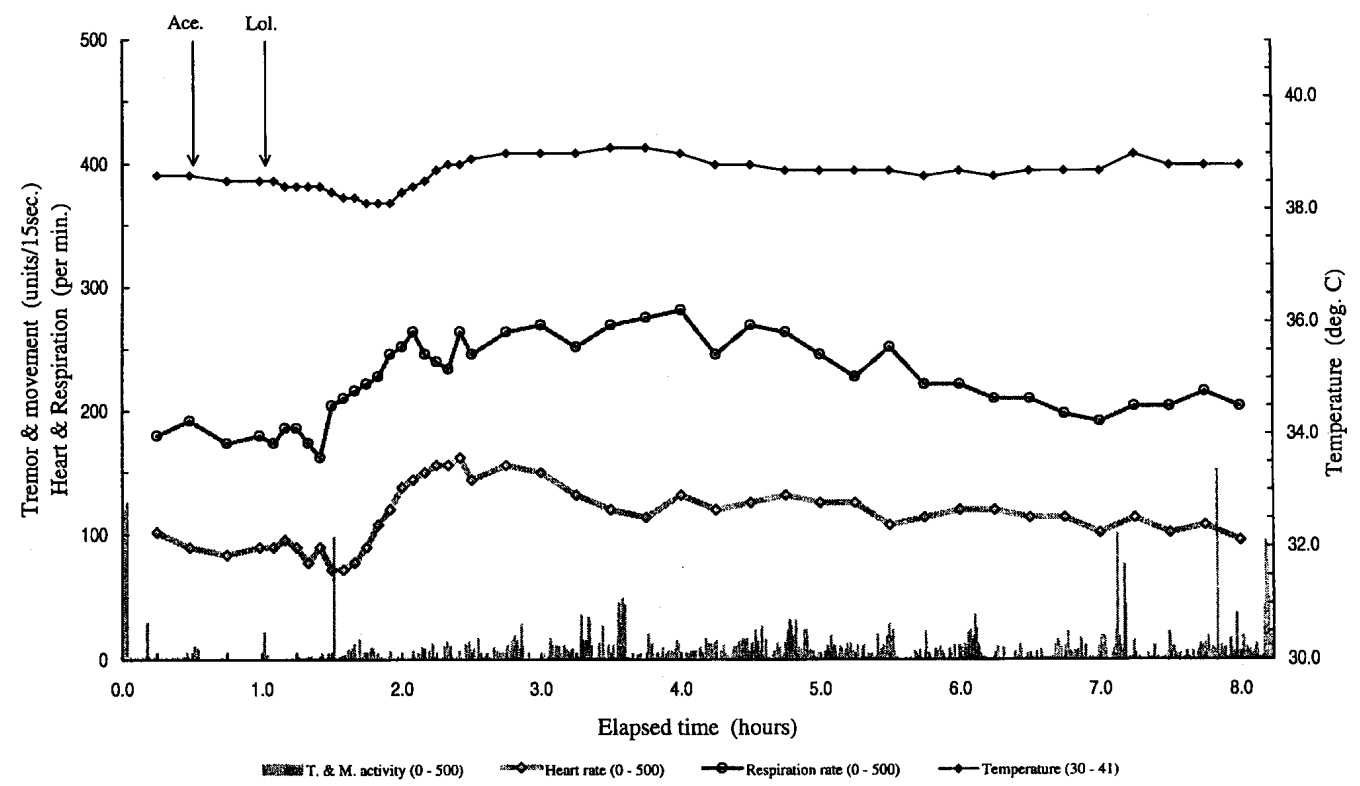

B

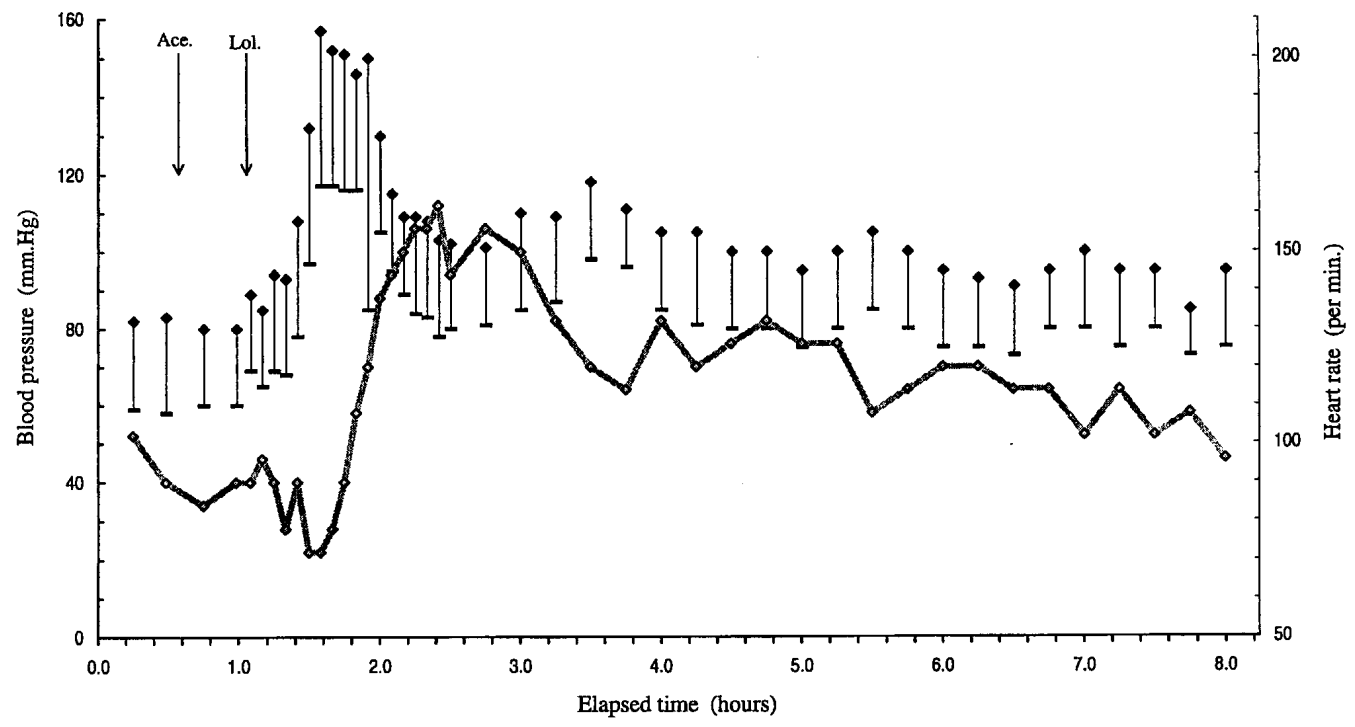

- Systolic BP $(0-160) \quad$ - Diastolic BP $(0+160) \quad-$ Heart rate $(50$ - 210) 
The excitatory responses remaining after atropine demonstrate other mechanisms exist and these also may involve high-conductance calcium-activated $\mathrm{K}^{+}$channels (McLeay et al. 1999). These channels have been shown to cause hyperpolarisation and relaxation of smooth muscle (Braydon \& Nelson 1992; Nelson et al. 1995). Blockage of these channels by a number of toxins including paxilline and the scorpian toxins charybotoxin and iberiotoxin may account for excitatory responses of these substances on smooth muscle preparations (Carl et al. 1996; DeFarias et al. 1996; Toro et al. 1998).

The inhibitory effects of paxilline and lolitrem B on the cyclical motility of the reticulorumen may arise through a number of pathways (McLeay et al. 1999). These contractions are stimulated by excitation of vagal efferent fibres originating from gastric centers in the medulla oblongata of the brain, which in turn are excited or inhibited by afferent stimulation from many areas of the gut, including the reticulorumen itself (Harding \& Leek 1971; Titchen 1968). Reflex inhibition of motility results from pharmacologically-induced increased local activity of the reticulorumen (Ruckebusch 1989). Increased intrinsic activity was a feature of the excitatory effects of paxilline and lolitrem $B$ on the reticulum and rumen in our experiments and may have been responsible for the inhibitory effects on cyclical contractions.

While this may be one possible route for the inhibition, direct effects on the gastric centres in the brain should not be ruled out. Tremorgens have been associated with pathological lesions of the cerebellum of the brain of sheep (Munday \& Mason 1967; Mason 1968; Cavanagh et al. 1998), and the occurrence of nystagmus in sheep after the administration of paxilline and lolitrem B demonstrated a central action of these mycotoxins in our experiments (McLeay et al. 1999). The possibilty of a direct action on the gastric centres or other higher centres warrants further study.

The responses in blood pressure and heart rate to paxilline and lolitrem $B$ indicate the initial effect was on peripheral vasoconstriction, followed by increased heart rate. Vasoconstriction may have arisen through blockade of high-conductance calcium-activated $\mathrm{K}^{+}$ channels, as these have been shown to regulate smooth muscle tone of arteries, their activation causing relaxation (Braydon \& Nelson 1992; Nelson et al. 1995). Increases in heart rate occurred at a time when blood pressure was dropping from its maximal level (see Figure 2B), but was still above basal levels and thus was unlikely to be a reflex response to lowered blood pressure. Generally, increases in heart rate, respiration rate and body temperature were closely associated with tremoring and included discrete changes in association with the "clonal tremors" (groups of tremors interspersed with quiescent periods) of lolitrem $\mathrm{B}$, for example 7 hours after its dosing (see Figure 2A). These responses were probably indirect, reflecting the greater oxygen demands and heat production resulting from the increased skeletal muscle activity of tremoring. Respiration rate (panting) is an important mechanism whereby sheep control body temperature. An initial drop in temperature after lolitrem B administration had a similar time course to the rise in blood pressure (see Figure $2 \mathrm{~A} \& \mathrm{~B}$ ), and may have been due to peripheral vasoconstriction reducing blood flow to the region in which the thermistor was located.

In summary, these experiments have shown that as well as effecting tremoring and producing the well known staggers-syndrome, paxilline and lolitrem $B$ have widespread effects on other physiological activities. Their effects on smooth muscle of the gut are likely to have serious consequences for digestion in animals ingesting these tremorgenic mycotoxins. Evidence of impaired digestion of animals grazing endophyteinfected pastures has been reported as scouring, dag formation and ill thrift (Fletcher 1993). While our present experiments have investigated effects on smooth muscle of the gut, one likely mechanism of action of the mycotoxins involving ion channels in cell membranes and their effects on neurotransmitter release and action, suggests it is highly probable they also affect the secretory activities of the gut, which together with alterations in motility would contribute to clinical features such as scouring. It is also likely that other mycotoxins present in pasture, such as the ergot-related toxins which have well known vascular effects, may exacerbate the situation. We are currently extending our research in sheep to investigate the effects of the ergopeptides associated with endophytes.

\section{ACKNOWLEDGEMENTS}

The authors thank Margaret Auger for the care of the animals, Dr Chris Miles and Dr Sarah Munday-Finch for mycotoxins, Dr Gordon Reynolds for catheterisation of femoral arteries in the sheep, and Peter Embling for assistance in the preparation of figures.

\section{REFERENCES}

Braydon, J.E.; Nelson, M.T. 1992. Regulation of arterial tone by activation of calcium-dependent potassium channels. Science 256: 525-535.

Carl, H.; Lee, H.K.; Sanders, K.M. 1996. Regulation of ion channels in smooth muscle by calcium. American Journal of Physiology 271: C9-C34.

Cavanagh, J.B.; Holton, J.L.; Nolan, C.C.; Ray, D.E.; Naik, J.T.; Mantle, P.G. 1998. The effects of the 
tremorgenic mycotoxin penitrem $\mathrm{A}$ on the rat cerebellum. Veterinary Pathology 35:53-63.

DeFarias, F.P.; Carvalho, M.F.; Lee, S.H.; Kaczorowski, G.J.; Suarez-kurtz, G. 1996. Effects of the $\mathrm{K}^{+}$channel blockers paspalitrem-C and paxilline on mammalian smooth muscle. European Journal of Pharmacology 314: 123-128.

Fletcher, L.R. 1993. Grazing ryegrass/endophyte associations and their effect on animal health and performance. pp. 115-120. In: Proceedings of the Second International Symposium on Acremonium/ Grass Interactions.

Gallagher, R.T.; Hawkes, A.D.; Steyn B.S.; Vleggaar, R. 1984. Tremorgenic neurotoxins from perennial ryegrass causing ryegrass staggers disorder of livestock: Structure elucidation of Lolitrem B, Journal of the Chemical Society, Chemical Communications. London, 614-616.

Gallagher, R.T.; White, E.P.; Mortimer, P.H. 1981. Ryegrass staggers: isolation of potent neurotoxins lolitrem A and lolitrem B from staggers-producing pastures. New Zealand Veterinary Journal 29: 189-190.

Harding, R.; Leek, B.F. 1971. The locations and activities of medullary neurones associated with ruminant forestomach motility. Journal of Physiology 219: 587-610.

Hawkes, A.D.; Embling, P.P; Towers, N.R. 1993. Breeding for resistance to ryegrass staggers. New Zealand Veterinary Journal 41: 217-218.

Knaus, H-G.; McManus, O.B.; Lee, S.H.; Schmalhofer, W.A.; Garcio-Calvo, M., Helms, L.M.H.; Sanchez, M.; Giangiacomo, K.; Reuben, J. P.; Smith, A. B. III; Kaczorowski, G.J.; Garcia, M. L. 1994. Tremorgenic indole alkaloids potently inhibit smooth muscle high-conductance calcium-activated potassium channels. Biochemistry 33: 5819-5828.

Mason, R.W. 1968. Axis cyclinder degeneration associated with ryegrass staggers in sheep and cattle. Australian Veterinary Journal 44: 428.

McLeay, L.M.; Comeskey, M.A.; Waters, M.J. 1990. Effects of epidermal growth factor on gastrointestinal electromyographic activity of conscious sheep. Journal of Endocrinology 124: 109-115.

McLeay, L.M.; Smith, B.L.; Munday-Finch, S.C. 1999. Tremorgenic mycotoxins paxilline, penitrem and lolitrem B, the non-tremorgenic 31-epilolitrem B and electromyographic activity of the reticulum and rumen of sheep. Research in Veterinary Science 66: 119-127.

Munday, B.L.; Mason, R.W. 1967. Lesions in ryegrass staggers in sheep. Australian Veterinary Journal 43: 598-599.

Munday-Finch, S.C.; Miles C.O.; Wilkins, A.L.; Hawkes, A.D. 1995. Isolation and structure elucidation of lolitrem A, a tremorgenic mycotoxin from perennial ryegrass infected with Acremonium lolii. Journal of Agricultural and Food Chemistry 43: 1283-1288.

Nelson, M.T.; Cheng, H.; Rubart, M.; Santana, L.F.; Bonev, A.D.; Knot, H.J.; Lederer, W.J. 1995. Relaxation of arterial smooth muscle by calcium sparks. Science 270: 633-637.

Penn, J.; Mantle, P.G. 1994. Biosynthetic intermediates of indole-diterpenoid mycotoxins from selected transformations at C-10 of paxilline. Phytochemistry 35: 921-926.

Robitallie, R.; Charlton, M.P. 1992. Presynaptic calcium signals and transmitter release are modulated by calcium-activated channels. Journal of Neuroscience 21: 297-305.

Ruckebusch, Y. 1989. Gastrointestinal motor functions in ruminants. pp. 1225-1282. In: Handbook of Physiology. The Gastrointestinal System. Section 6, vol. I. Eds. Schultz, S.G.;Wood J.D.; Rauner, B.B. Oxford University Press. New York.

Selala, M.I.; Laekeman, G.M.; Loenders, B.; Musuku, A.; Herman, A.G.; Schepens, P. 1991. In vitro effects of tremorgenic mycotoxins. Journal of Natural Products 54: 207-212.

Smith, B.L.; McLeay, L.M.; Embling, P.P. 1997. Effects of the mycotoxins penitrem, paxilline and lolitrem $\mathrm{B}$ on the electromyographic activity of skeletal and gastrointestinal smooth muscle of sheep. Research in Veterinary Science 62: 111-116.

Stern, P.S.; Vleggaar, R. 1985. Tremorgenic mycotoxins. pp. 1-80. In: Progress in the Chemistry of Organic Natural Products. Vol. 48. Eds. Herz, W.; Grisebach, H.; Kirby, G.W.; Tamm, Ch. SpringerVerlag, New York.

Stretton, D.; Miura, M.; Belvisi, M.G.; Branes, P.J. 1992. Calcium activated potassium channels mediate prejunctional inhibition of peripheral sensory nerves. Proceedings of the National Academy of Sciences USA 89: 1325-1329.

Toro, L.; Wallner M.; Meera, P.; Tanaka Y. 1998. Maxi$\mathrm{K}_{\mathrm{Ca}}$, a unique member of the voltage-gated $\mathrm{K}$ channel superfamily. News in Physiological Sciences 13: 112-117.

Titchen, D.A. 1968. Nervous control of motility of the forestomach of ruminants. pp. 2705-2724. In: Handbook of Physiology. Alimentary Canal. Bile; Digestion; Ruminal Physiology. Section 6, Volume V. Ed. Code, C.F. American Physiological Society. Washington DC.

Weedon, C.M.; Mantle, P.G. 1987. Paxilline biosynthesis by Acremonium lolii; a step towards defining the origin of lolitrem neurotoxins. Phytochemistry 26: 969-971. 
